\title{
Biochip based on arrays of switchable magnetic nano-traps
}

ChristopherVautrin $^{\mathrm{a}, \mathrm{c}}$, Maria Vyazmensky ${ }^{\mathrm{e}}$, Stanislav Engel ${ }^{\mathrm{b}, \mathrm{e}}$, Stephan Mc. Murtry ${ }^{\mathrm{a}}$, Michel Hehn $^{\text {a, }}$, François Montaigne ${ }^{\mathrm{a}}$, Daniel Lacour ${ }^{\mathrm{a}}$, and Robert S. Marks ${ }^{\mathrm{c}, \mathrm{d}, \mathrm{e}, \mathrm{f}, \mathrm{g}}$

${ }^{a}$ Institut Jean Lamour, CNRS - Nancy-Université - UPV-Metz, Boulevard des Aiguillettes BP 70239, F-54506 Vandoeuvre lès Nancy, France.

${ }^{b}$ Department of Clinical Biochemistry and Pharmacology, Faculty of Medicine, Ben-Gurion University of the Negev, Beer-Sheva, 84105, Israel

${ }^{c}$ Department of Biotechnology Engineering, Faculty of Engineering Science, Ben-Gurion University of the Negev, Beer-Sheva, 84105, Israel

${ }^{d}$ School of Material Science and Engineering, Nanyang Technology University, Nanyang Avenue, 639798, Singapore

${ }^{e}$ National Institute for Biotechnology in the Negev, Ben-Gurion University of the Negev, Beer-Sheva, 84105, Israel

${ }^{f}$ The Ilse Katz Center for Meso and Nanoscale Science and Technology, Ben-Gurion University of the Negev, Beer-Sheva 84105, Israel

* Corresponding author: Michel Hehn

Michel.hehn@univ-lorraine.fr 


\begin{abstract}
$\underline{\text { Abstract }}$
Magnetic biochips based on switchable magnetic nano-traps were developed and optimized so as to enable faster bio-assays via a reduction of steps and the efficient capture of analytes in a sample test solution. Nano-traps are based on snake shaped magnetic nanowires made by microelectronic technics from a thin magnetic layer. This optimized design ensures high local fields when the trap is activated and reduced stray fields when deactivated with respect to previous designs. Our proof-of-concept model consists to determine the anchorage of synthetic peptides in a bilayer enveloping magnetic beads by revealing their presence via fluorescently labeled immunoglobulins elicited against the native proteins of the said exposed peptides.
\end{abstract}

Keyword: Magnetic domains walls, Magnetic Nano-traps, Bio-assay, Peptide, Magnetic beads. 


\section{I - Introduction}

Medical diagnostics is a concern that has grown with human progress [1]. The conventional leading medical diagnostics, such as standard ELISA and PCR, are not only sensitive but also affinity-based devices, which run a large part of our diagnostics nowadays. There have been attempts at increasing their diagnostic power by finding alternative ways to treat the sample (lab-on-a-chip), increasing sensitivity which could be done chemically (dendrons [2], and more), biologically (avidin-biotin scaffolds) or physically (metal enhanced fluorescence [3]). In the last years, a number of groups have attempted to increase the overall bioassay efficiency by creating biosensor-based devices using the same biological principles but by adding a transducer such as a fiber optic [4], [5], an electrode [6] or a piezoelectric crystal [7]. Reaching the target analyte faster and in an efficient manner, especially in complex media, is also of high interest. Indeed, in a conventional ELISA test in a microtiter well plate, the diffusion obstacle of the measured analyte to the solid phase surface, especially in the case of a complex medium, such as in whole blood or food processed within a stomacher bag is also a limiting factor. As a consequence, there is not only an increase of the overall time of the immunoassay, but also a decrease in the chance in collecting a sizable or even representative analyte population from the solution. This obstacle has found solutions in the development of additional and complementary tests, found in micro-fluidics incorporated ELISA [8], ELISA Reverse Based Assay [9] and Bead Based ELISA [10]. The use of magnetic beads in order to create a suspension which increases the statistical probability of interaction between the solid phase (the bead) and the target molecules has shown itself to be a very successful method in many studies [11].

Magnetic beads (from several nanometers up to hundreds of microns) are usually spherical particles doped with iron oxide. Depending on their size, they show paramagnetic or superparamagnetic properties, enabling them to interact with magnetic fields. 
When surface-functionalized, microbeads can interact on one hand with cells or biomolecules in lab-on-chip devices and on the other hand with local magnetic fields enabling nanoscale control of position. Different strategies have been used to create local fields and manipulate beads like microcoils or electromagnets with or without magnetic circuit made of magnetic wires to concentrate the magnetic flux $[12,14,15,16,26]$, magnetic nanostructures and rotating applied field $[13,21,20]$ or magnetic nanowires hosting domain walls $[17,18,19,22$, 24] but none of these present the capabilities of the nano-trap designed and tested in our study. First of all, our trap can be deactivated as for electromagnets but does not use power as for electromagnets. The activation of the trap can be made using only a permanent magnet that makes the device wireless, transportable, weight less and working without any powersupply. When nanostructures are used, it is not possible to deactivate the traps with dots or the deactivation is limited with domain walls in zig zag configuration. Therefore they are efficient only to move beads on a surface. Second, our trap could be able to locate the beads on the surface of the device as for electromagnets with localized electrodes while beads agglomerate on the periphery of the device or on the nanostructures leading to a reduced interaction between the biologic sample to be tested and the excitation used for the test (for example the light).

The new magnetic biochip based on switchable magnetic nano-traps was developed and optimized so as to enable fast bio-assays via a reduction of steps and the efficient capture of analytes in a sample test solution. Nano-traps are based on snake shaped magnetic nanometer wires made by microelectronic techniques from a magnetic full film. This optimized design ensures high local field when activated and reduced stray field when deactivated with respect to previous designs. Our proof-of-concept model consists to determine the anchorage of synthetic peptides in a bilayer enveloping magnetic beads by revealing their presence via fluorescently labeled immunoglobulins elicited against the native proteins of the said exposed peptides. The resulting fluorescence produced at the end of the immunoassay is measured here by fluorescent microscopy. The use of a microscope allows us to check that the beads are indeed captured at localized focal points and that the coated marker fluorescence can be individually (or in several bead aggregates) distinguished.

\section{II - Material and methods}

\section{Biochip coated with ferromagnetic tracks}

The magnetic tracks have been patterned from a multilayer thin film stack deposited on a glass substrate by sputtering. The multilayer stack structure is the following: Glass//Ta(5)/Co(40)/ $\mathrm{Pt}(3)$ (numbers in bracket are thicknesses in $\mathrm{nm}$ ). The Ta buffer layer is used to ensure both the adhesion of the multilayer on the glass substrate and a low surface roughness. The Pt capping layer is used to prevent the oxidation of the Co layer. The tracks are then designed by electron beam lithography and transferred into the Co layer using dry $\mathrm{Ar}^{+}$etching. Two different designs for the magnetic tracks have been tested and are presented in Fig. 1. The first design is made of straight corners (called zigzag) as used in previous studies $[17,18,19,24]$ while the other has curvy features (called snake). Each design covers a squared surface of $200 \times 200 \mu \mathrm{m}^{2}$. 


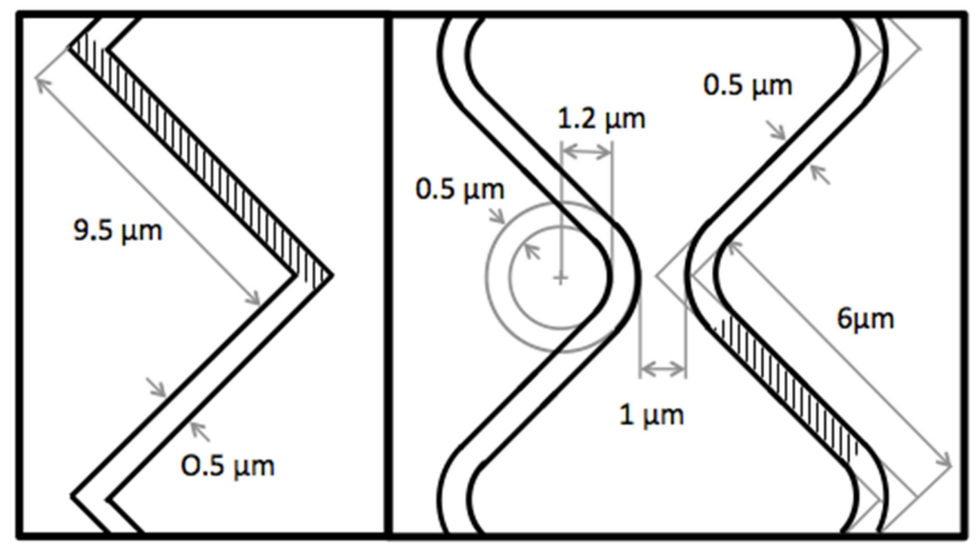

Fig. 1. Scheme of the two geometries of magnetic tracks tested in our study. Left: Zigzag. Right: Snake.

2. Creation of the magnetic domains walls

A one step protocol is required to create magnetic domains walls in the magnetic tracks. The generation or annihilation of the magnetic domain walls is done by a simple exposure to a $\mathrm{NdFeB}$ permanent magnet of volume $1.5 * 0.8 * 0.2 \mathrm{~cm}^{3}$ during a time less than 1 second. The field, of intensity around 100 Gauss, is strong enough to align the magnetization along its direction. Then, generation or annihilation of the magnetic domain walls will depend on the direction of the applied field. The tracks have been designed in such a way that their aspect ratio imposes a so-called shape anisotropy. Consequently the stable position for the magnetization in absence of the external magnetic field is along the tracks borders.

To create the domain walls, a magnetic field (denoted $\mathrm{H}$ in Fig. 2) is applied perpendicularly to the track's long axis. Therefore the stray field of the permanent magnet is oriented perpendicularly to the tracks long axis when approaching the magnet. Thus, the tracks magnetization is saturated in the direction of the external field (see Fig. 2(a)). Thereafter, reducing the magnetic field to zero (i.e. taking the permanent magnet away) leads to a progressive rotation of the magnetization towards its stable positions, as seen in Fig. 2 (b). Finally when the permanent magnet is totally removed $(\mathrm{H}=0)$, the magnetization is aligned along the track and creates magnetic domain walls located at the kinks (see Fig. 2 (c)). From now, in the case of tracks hosting domain walls, it will be referred as the "Trap ON" case. 


\section{Trap ON procedure}

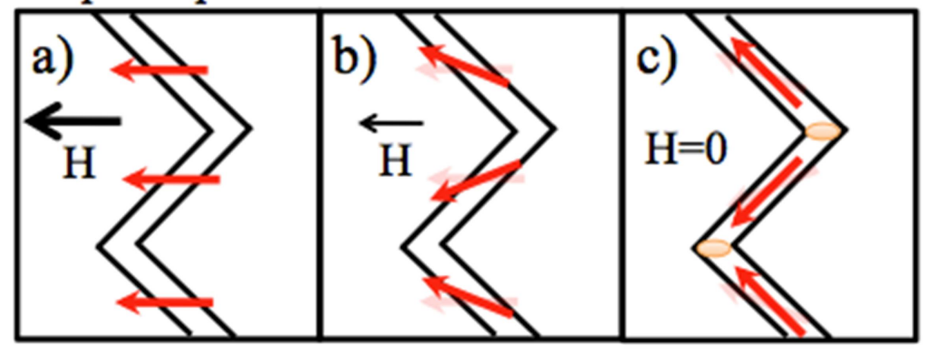

Trap OFF procedure

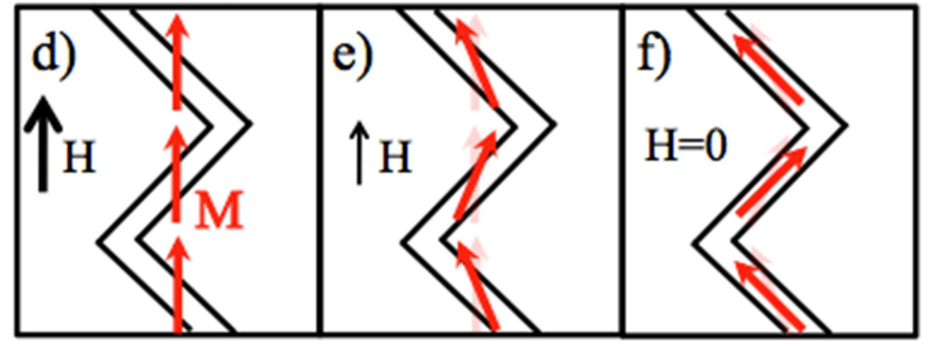

Fig. 2: Sketches of the creation (trap ON case) and annihilation (trap OFF) procedures of magnetic domain walls.

To annihilate the domain walls a very similar procedure is followed. This time the permanent magnet has just to be turned $90^{\circ}$ in order to apply its stray field in the direction of the tracks long axis and thus the magnetization is likewise turned (Fig. 2 (d)). Thereafter, as the permanent magnet is removed, a reduction of the magnetic field occurs with the consequent progressive rotation of the magnetization towards its stable positions (Fig. 2 (e)).With this field configuration, at the end of the procedure, no domain wall is present. In the following parts of this paper, the case of tracks without domain walls will be referred as the "Trap OFF" case.

\section{The magnetic beads}

The model beads used herein were magnetized liposomes formed by coating magnetic beads with a lipid bilayer in which synthetic palmitoylated peptides carrying a specific epitope of an HIV binding receptor CCR5 were incorporated. Their presence will be revealed here by a specific fluorescently-labeled immunoglobulin. Our negative control experiment consists of the aforementioned magnetic liposomes without the incorporation of the said peptide. Thus, one expects a lack of molecular recognition between the specific immunoglobulins and a surface lacking the corresponding epitope. As a result, the antibodies will not bind to the beads and thus they will not exhibit any fluorescence assuming that the assay will not suffer from non-specific binding of the marker immunoglobulin. These steps are detailed as follow.

\section{Preparation of liposomal solutions:}

The synthetic lipids were obtained as chloroform solutions from Avanti Polar Lipids (Alabaster, AL, USA). A total of $10 \mathrm{mg}$ of chloroform-dissolved lipids (1-palmitoyl-2-oleoylsn-glycero-3-phosphocholine (POPC), 1-palmitoyl-2-oleoyl-sn-glycero-3phosphoethanolamine (POPE), and dimyristoylphosphatidic acid (DMPA)), mixed in a molar 
ratio of 6:3:1, were dried under vacuum. One milliliter of PBS (Phosphate-Buffered Saline, a buffer solution commonly used in biological research) obtained from Hylabs (Rehovot, Israel) was added, and a liposomal solution was then obtained by a 10 min ultrasonication at room temperature in a bath sonicator (ELMA S-30H, Singen, Germany). A liposomal solution of the head group-modified synthetic lipid 1,2-dioleyl-sn-glycero-3-phosphoethanolamine-n(biotinyl) (Biotinyl-DOPE) at a final concentration of $1 \mathrm{mg} / \mathrm{ml}$ was prepared separately using the same protocol. All liposomalsolutions were kept at $-80{ }^{\circ} \mathrm{C}$ until use.

\section{Preparation of magnetic liposomes:}

Approximately $1.7 \times 10^{8}$ streptavidin-coated beads $\quad(1 \quad \mu \mathrm{m} \quad$ silica-coated superparamagnetic beads, BcMag'TM, BioClone Inc., San Diego, CA,) were washed twice with PBS and combined with $1 \mathrm{mg}$ of liposomes composed of the aforementioned lipid mixture and $10 \mu \mathrm{g}$ of liposomes made from Biotinyl-DOPE. The mixture was solubilized in $1 \mathrm{ml}$ of solubilization buffer $\left(100 \mathrm{mM}(\mathrm{NH} 4)_{2} \mathrm{SO}_{4}, 20 \mathrm{mMTris}-\mathrm{HCI}(\mathrm{pH} 7.5), 10 \%(\mathrm{v} / \mathrm{v})\right.$ glycerol, $1 \%$ (w/v) CHAPSO) andincubated at $4^{\circ} \mathrm{C}$ for $1 \mathrm{~h}$. After incubation, the detergent was slowly removed by dialysis for $24 \mathrm{~h}$ at $4^{\circ} \mathrm{C}$ against $100 \mathrm{mM}\left(\mathrm{NH}_{4}\right)_{2} \mathrm{SO}_{4}, 20 \mathrm{mMTris}-\mathrm{HCI}(\mathrm{pH} 7.5)$, and 10\% (v/v) glycerol, using a $10 \mathrm{kDa}$ cutoff membrane (Slide-A-Lyzer $10 \mathrm{~K}$; Pierce, Rockford, IL). The resulting magnetic liposomes were washed twice with PBS and stored in an eppendorf tube in PBS at $4^{\circ} \mathrm{C}$ until use.

Preparation of magnetic peptidoliposomes:

The peptide corresponding to the N-terminus portion of the human CCR5 receptor (amino acids 1-27) was palmitoylated at the C-terminus (GL Biochem Ltd., Shanghai, China). $50 \square \mathrm{g}$ of the palmitoylated peptide (1:100 peptide/total lipids molar ratio) were added as a $1 \mathrm{mg} / \mathrm{ml}$ ethanol solution to $1 \mathrm{mg}$ lipid mixture (as described previously) prior to the addition of the solubilization buffer, and the magnetic liposomes were prepared as previously described.

Antibody binding to peptidoliposomes:

In order to demonstrate that the peptides in the peptidoliposomes were available for binding to the marker immunogobulins and therefore positioned as predicted, approximately $0.3 \times 10^{8}$ liposomes were prepared in the presence (peptidoliposomes) or absence (negative control) of the CCR5-derived peptide and these were suspended in $0.5 \mathrm{ml} 2 \%$ (v/v) fetal calf serum (FCS) in PBS, and incubated for $1 \mathrm{~h}$ at room temperature in the presence of $2 \square \mathrm{g}$ of PE-conjugated anti-human CD195 (CCR5 N-term, BioLegend,BLG-321405, San Diego, CA) antibody. These solutions were then tested by applying them directly onto the magnetic chip surfaces.

III - $\underline{\text { Results and discussion }}$

\section{Demonstration of Trap ON and Trap OFF states}

For this demonstration, the surface of our biochip was scanned using a near field microscope 3100 Dimension from Veeco. The microscope was used in the magnetic force mode (MFM) [23]. Two scans are performed. During the first scan the topography of the surface is recorded in the tapping mode while during the second scan a phase image is recorded at a constant height of $150 \mathrm{~nm}$. During this second scan the microscope allows to obtain images having a phase contrast proportional to the second derivate of the sample stray field component normal 
to the surface. So, locations where magnetic stray fields escape from the tracks can be identified. The recorded images as well as the associated simulations of the magnetic configuration in the zigzag shaped tracks are shown in Fig. 3.

In a first step, the magnetic trap was set $\mathrm{ON}$ following the previously described procedure. The recorded MFM image is shown in Fig. 3(d). Areas having dark and white contrasts are clearly visible. Both of these contrasts are located as expected at the kinks of the track [24]. In order to clarify the distribution of magnetization, the process was simulated with the MUMAX code [25], using a cell size of $10 \mathrm{~nm}$ and the following parameters corresponding to the Co ferromagnetic material (the material used for fabrication of the traps) : saturation magnetization equal to $1400.10^{3} \mathrm{~A} \cdot \mathrm{m}^{-1}$, exchange constant $=30.10^{-12} \mathrm{~J} \cdot \mathrm{m}^{-1}$ and thickness of $40 \mathrm{~nm}$.
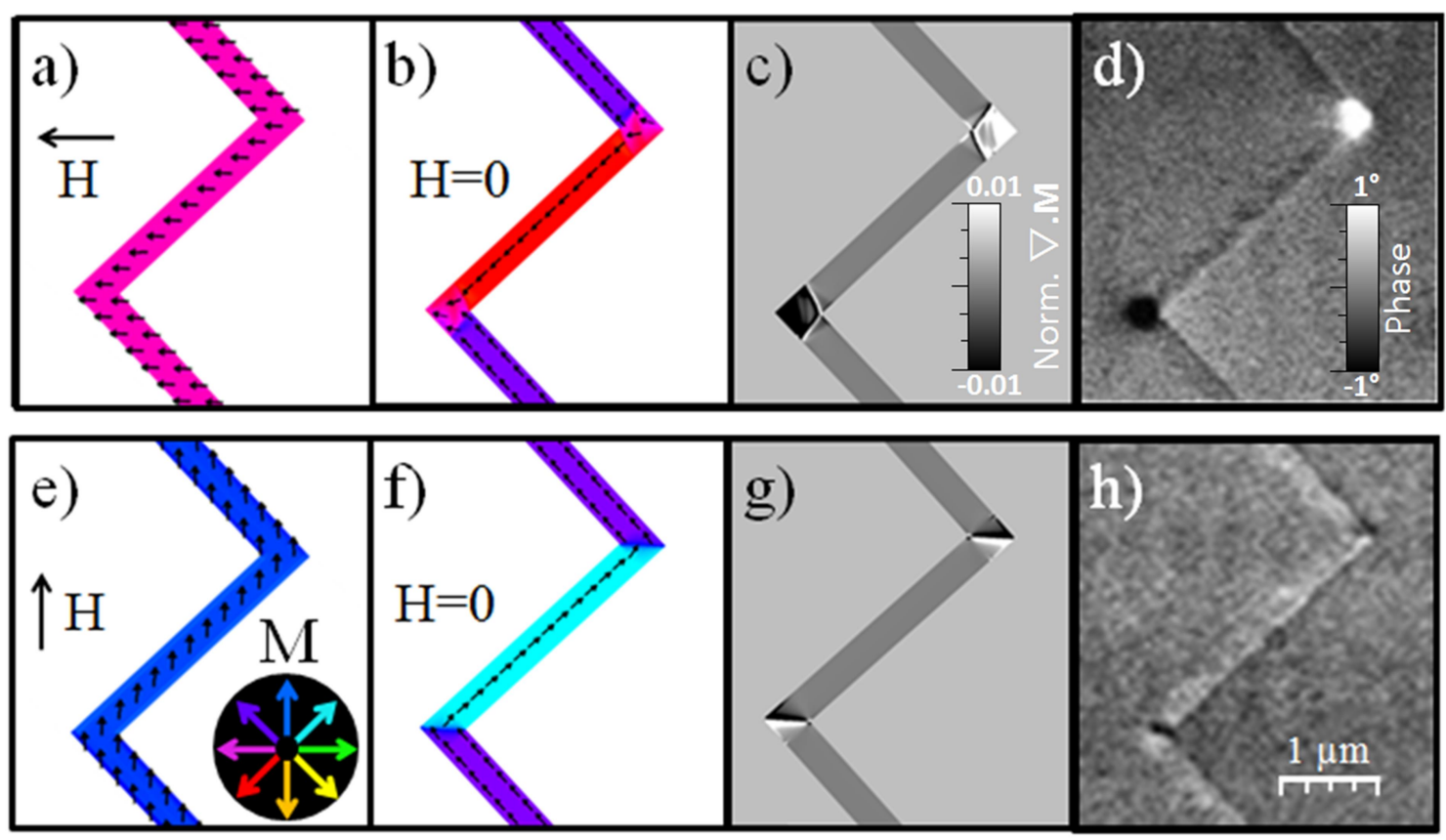

Fig. 3. Test of the trap ON and trap OFF procedures. (d) and (h): MFM images of the magnetic track after the application of the trap ON (d) and trap OFF (h) procedures. (a) and (e) simulation of the magnetization in the track with a saturating magnetic field applied perpendicularly (a) and along the track (e) long axis. (b) and (f) simulations of the magnetic configuration when the magnetic field is reduced to zero after perpendicular (b) and parallel (f) saturation. (c) and (g) calculated normalized divergence of magnetization $(\nabla . M)$ from the simulations presented in (b) and (f).

In a first step, the magnetization is saturated (fully aligned) with a field applied perpendicularly to the long track axis (Fig. 3(a)). Then, when the field is brought to zero, the magnetization mostly aligns with the border of the track. One should notice that the two magnetic domain walls are located at the kinks of the track. The top one is of tail-to-tail domains wall type with the magnetization of each surrounding domains pointing away from the wall, while the bottom one is a head-to-head wall with the magnetization of each surrounding domains pointing towards the wall. To fully understand the experimental MFM contrasts, the magnetic configurations and the magnetization divergence, which is a source of stray field probed by the MFM, have been calculated. The result of the calculation is presented in Fig. 3(c). The divergence of magnetization appears to be equal to zero in the 
track, except at places where domain walls are located. Note that the divergence associated to the head-to-head and to the tail-to-tail domain walls are of opposite sign. This is in a good agreement with the contrast observed in MFM (compare Fig. 3(c) to Fig. 3(d)). The presented MFM images, as well as the simulations, attest of the success of the trap ON procedure.

Then, the trap OFF procedure was tested. The experimental results (MFM images) and associated simulations are presented in Fig. 3(e) and Fig. 3(h). It can be seen on the MFM image reported in Fig. 3(h), that a stray field persists even in the trap OFF case. Thus, even in the absence of domains walls, the divergence of the magnetization at the kink is not equal to zero. This is confirmed by the calculated divergence of magnetization presented in Fig. $\mathbf{3}(\mathrm{g})$. As this divergence leads to some stray field, this is problematic for our biochip in the OFF mode. Consequently the design of the magnetic tracks has to be improved. In order to decrease the divergence of magnetization which is the main source of stray field in our case, the radius of curvature has been increased and a snake shape of tracks has been chosen.

Experimental results as well as the corresponding simulations are presented in Fig. 4. As expected, the increase of curvature radius led to a strong reduction in the magnetization divergence in the Trap OFF. The absence of contrast on the MFM image can be noticed, Fig. 4 (c), as well as in the calculated divergence of magnetization, Fig. 4 (b). In the trap ON case, the presence of head-to-head and tail-to-tail domain walls was predicted by simulation (Fig. 4 (a)) and confirmed by MFM (Fig. 4 (b)).

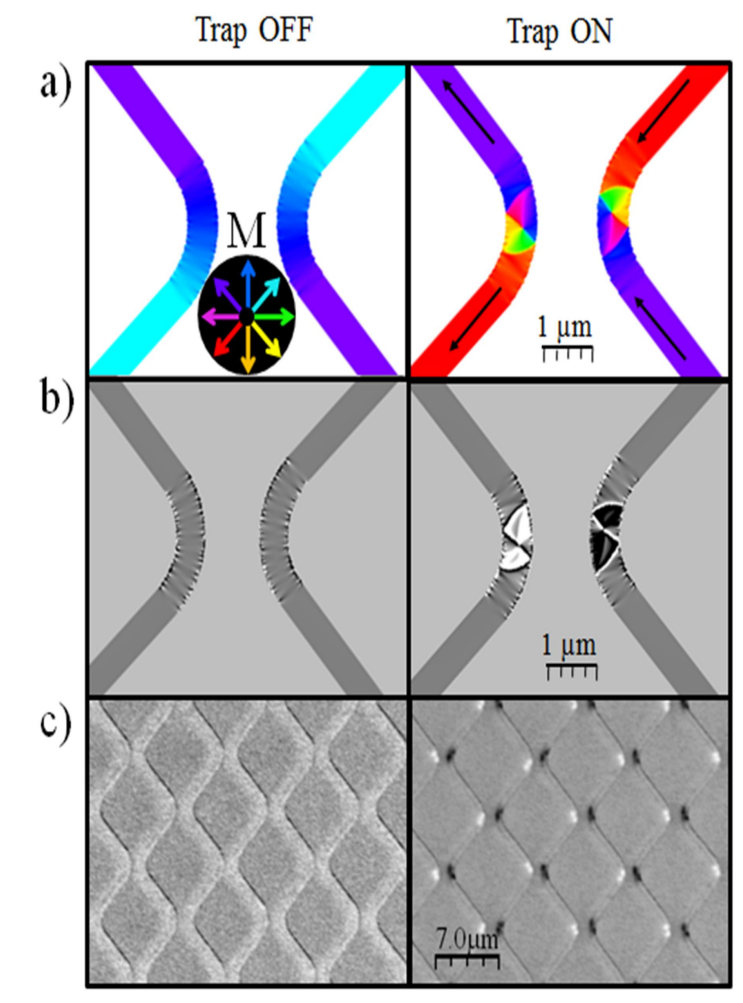

Fig. 4. Test of the trap OFF and trap ON procedures with an improved design of the magnetic tracks. Row (a) simulations of the magnetic configurations when the magnetic field is reduced to zero after application of the trap OFF and the trap ON procedures. Row (b) divergence of the simulated magnetic configuration presented in a). Both cases: OFF and ON cases are resented. (c) MFM measurements of the magnetic tracks after application of the trap OFF and trap ON procedures. 


\section{Test of nano-trap efficiency}

The aim of our study consists to create a magnetic biochip with the capability to trap beads which have captured the analyte of interest and then to measure its presence via a luminescent marker (chemiluminescent, bioluminescent, or fluorescent depending on the bioassay system used) whose radiation can be easily coupled to a chip. To check the efficiency of the magnetic nano-traps, a drop of distilled water containing commercial $500 \mathrm{~nm}$ diameter magnetic beads (hhtp://www.micromod.de) was deposited onto the chip with either the trap ON or the trap OFF configuration. One minute after the drop deposition, the biochip surface was rinsed with distilled water. Images of the surface were then recorded with an optical microscope (Fig. 5) with both states of the magnetic trap. These two images clearly show that in the trap OFF state no magnetic nano-beads are present (Fig. 5 (a)) while in the trap ON case (Fig. 5 (b)), nano-beads were found at the curvatures of the magnetic tracks as expected.

a) Trap OFF

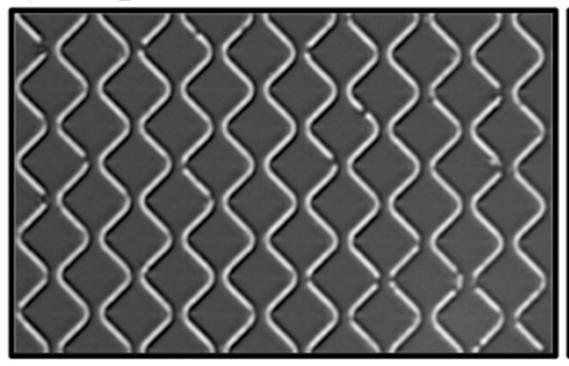

b) Trap ON

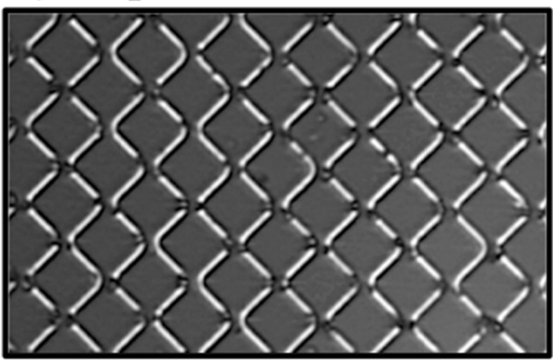

Fig. 5. Optical pictures of the chip surface after deposition of a drop of distilled water containing magnetic nano-beads followed by a wash with distilled water in (a) the trap OFF mode, (b) the trap ON mode.

In summary, we have shown that the magnetic beads are indeed trapped by the domain walls present in the magnetic tracks in the ON mode and do resist a water rinse. On the other hand, when the chip is in the OFF mode, the beads are easily removed by a simple water rinse. This experiment thus shows that the attractive forces are strong enough to resist the action of the water rinse, an obligatory step in bioassays such as that described hereafter.

\section{Model immunoassay based on the magnetic traps}

At first, the trapping efficiency of magnetic domain walls to retrieve magnetic liposomes from the solution was checked. A palmitoylated peptide containing an epitope recognized by the paratope of a specific fluorescently-labeled antibody was previously incorporated into the lipid bilayer. The beads were first incubated with the peptide-specific antibody and the mixture was placed onto the chip surface in the trap ON mode and the surface washed thrice with a slow flow of PBS on the surface (around $100 \mu \mathrm{lBS}$ ).

Three cleaning steps were sufficient to remove the beads throughout the chip except where the domain walls were located (Fig. 6).

The presence of the lipid bilayer on the surface of the magnetic beads did not interfere with the efficiency of the trapping, indicating that the magnetic field is sufficiently strong to be able to properly handle biologically or chemically modified magnetic particles. 
a) Trap ON with peptide

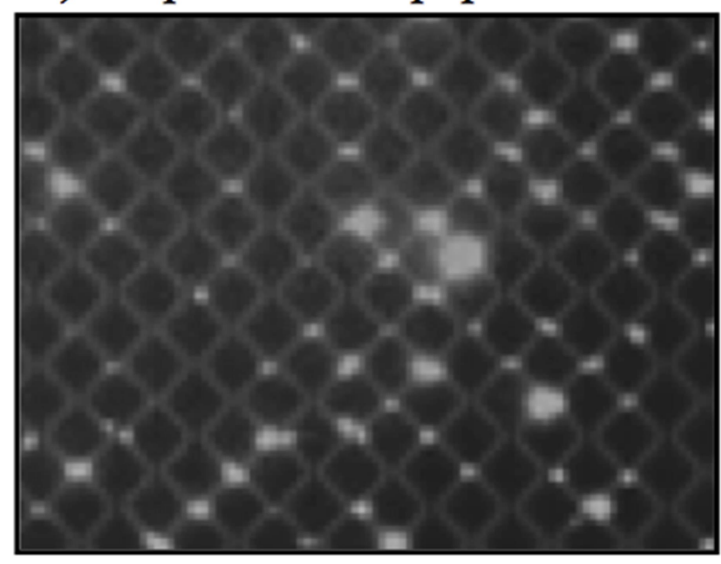

b) Trap ON without peptide

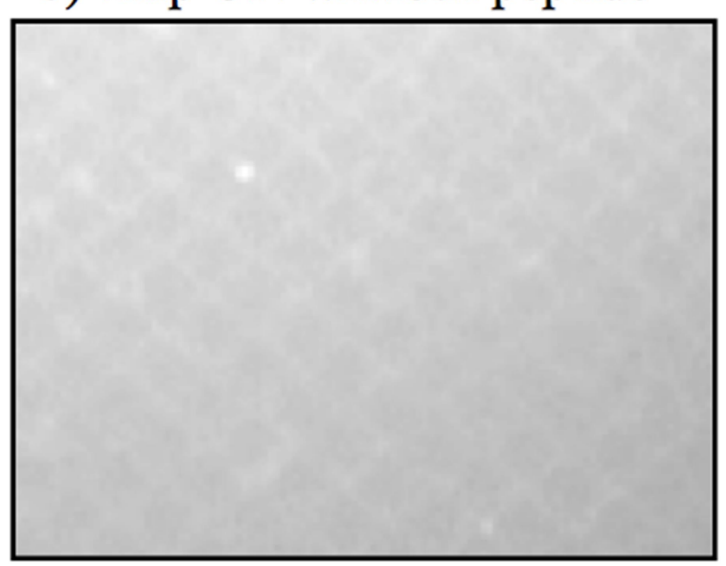

Fig. 6. Fluorescent pictures of the chip. (a) Trap ON; fluorescent particles on the surface the sample. Exposure time for the camera: $0.3 \mathrm{~s}$. The larger particles are aggregates of magnetic particles. (b) Trap ON; no fluorescent particles on the surface. Exposure time: 15s.

The fluorescence signal was specific to the presence of the peptide in the trapped beads indicating that the peptide was properly oriented and exposed to the antibody in solution and that the level of nonspecific binding of the antibody to the chip surface was insignificant and did not interfere with detection (see Fig. 6). In the case with peptide, 118 active sites (traps) among the 255 presented a fluorescence signal (Fig. 6 (a)). Concerning the case without peptide, none of the active sites are emitting a fluorescence signal taking into account that the exposure time has been increased by a factor 50 to obtain the image shown in Fig. 6(b). Thus, blocking of the surface with an inert protein, such as albumin, was not required.

\section{a) Trap ON}

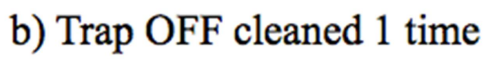

\section{c) Trap OFF cleaned 3 time}
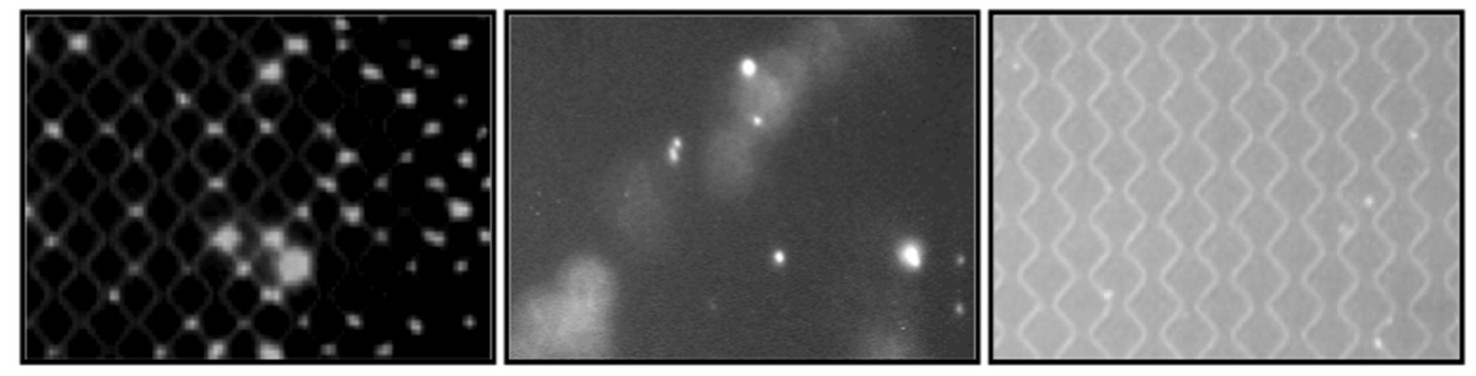

Fig. 7. Fluorescent pictures of the chip. (a) Trap ON; fluorescent particles on the sample. Exposure time for the camera: 0.3s. (b) Trap OFF; Fluorescent particles on the sample. Exposure time for the camera: 7s. For this picture, the chip was rinsed only one time.(c) Trap OFF; Fluorescent particles on the sample. Exposure time for the camera: 15s. For this picture, the chip was rinsed three times. 
The same experiment done without localized domain walls would have product aggregates that would have been difficult to quantify. So, using the 'trap-OFF' magnetic chips, beads would not be trapped, and therefore easily washed away (Fig. 7).

Finally, our test samples can easily be recovered by merely changing the magnetic field (Fig.7), which is sometimes important when working with rare biological samples, or if the biological material is needed for another analysis or experimental step, such as genetic engineering.

\section{IV - $\underline{\text { Conclusion }}$}

In this paper, a design of a magnetic trap based on magnetic nanowires with snake shape optimized for its OFF state is reported, a design that benefits from knowledge from the nanomagnetism community. The local field created by the domain walls at the snake corners and the absence of domain walls when the trap is deactivated ensures a strong attraction of the beads when the trap is activated while the device can be easily reset with one or two water cleaning for the next analysis. With respect to previous reports, our trap can be deactivated as for electromagnets but does not consum power as for electromagnets. The activation of the trap can be made using only a permanent magnet that makes the device wireless, transportable, weight less and working without any powersupply. In contrast, when nanostructures are used, it is not possible to deactivate the traps with dots or the deactivation is limited with domain walls in zig zag configuration.

From this first design, several evolutions can be forecasted since the magnetic wires can be miniaturized, down to $10 \mathrm{~nm}$ if needed using for example ebeam lithography. The decrease of the lateral size will decrease the field stray field and a compromise has to be found to attract single beads. Considering our results, the track parameters used in our study do not seem to be too far from the optimal ones to trap single beads. The snake can also be modified. First of all, the $6 \mu \mathrm{m}$ long part of the wire can be reduced if the shape anisotropy of the straight parts is conserved to align magnetization along the wire borders. Roughly, a factor of 5 should be conserved between the length of straight parts of the snake and the width of the wire. Then, in our demonstration, the distance between the snake corners has been set to $1 \mu \mathrm{m}$. This geometry does not seem to be optimal to locate beads between the corners. A study in which the distance is decreased should be done as an extension of this work. The stray field can be increased by buckling the stray field between the snake corners with opposite divergence. This could locate the beads on the glass substrate and increase the interaction between the biologic sample to be tested and the excitation used for the test (for example the light). 
Magnetic beads are used in biochips for bioassays that require a very effective separation of the target molecules from the complex media. However, due to their use, magnetic beads often easily aggregate after being subjugated to magnetic forces and require re-suspension. In our demonstration, aggregation seems to be limited and individual beads (or close to individual) are trapped meaning that future biological could lead to better quantified measurements. However, those measurements are beyond the scope of our paper dealing with the demonstration, on biological material, that our trap is efficient. Finally, as shown in this study, the preparation steps for biological analysis is reduced since 3 steps for analysis are needed and only one step to get the analysis device ready for another analysis.

\section{Acknowledgments}

We thank the various participants who have helped us including Dr. Levi Gheberand technicians who helped us conduct this project. This study was supported (in part) by grant no. 3-00000-7445 from the Chief Scientist Office of the Ministry of Health, Israel, by La Région Lorraine and Singapore NRF CREATE project on 'Nanomaterials for Energy and Water Management'. Experiments were carried out on IJL Project TUBE-Davms equipments funded by FEDER (EU), PIA (Programme Investissemnet d'Avenir), Region Grand Est, Metropole Grand Nancy and ICEEL. 


\section{$\underline{\text { References }}$}

[1] R. S. Marks, Dermatophytoses in Art, Journal of Medical and Veterinary Mycology (1991).

[2] O. Iliashevsky et al., Synthesis, characterization and protein binding properties of supported dendrons, Journal of Materials Chemistry 19 (2009) 6616.

[3] E. Eltzov et al., Metal-enhanced bioluminescence: An approach for monitoring biological luminescent processes, Applied Physics Letters 94 (2009) 083901.

[4] F. Cecchini et al., Chemiluminescent DNA optical fibre sensor for Brettanomyces bruxellensis detection, Journal of Biotechnology 157 (2012) 25.

[5] R. S. Marks et al., Chemiluminescent optical fiber immunosensor for detecting cholera antitoxin, Optical Engineering 36 (1997) 3258.

[6] K. Abu-Rabeah and R. S. Marks, Impedance study of the hybrid molecule alginatepyrrole: Demonstration as host matrix for the construction of a highly sensitive amperometric glucose biosensor, Sensors and Actuators B-Chemical 136 (2009) 516.

[7] G. Garai-Ibabe et al., Label free and amplified detection of cancer marker EBNA-1 by DNA probe based biosensors, Biosensors \& Bioelectronics 30 (2011) 272.

[8] A. Bange, H. B. Halsall and W.R. Heineman, Microfluidic immunosensor systems, Biosensors \& Bioelectronics 20 (2005) 2488.

[9] S. Folloni et al., Development of an ELISA Reverse-Based Assay to Assess the Presence of Mycotoxins in Cereal Flour, Food Analytical Methods 4 (2011) 221.

[10] N. Scholler et al., Bead-based ELISA for validation of ovarian cancer early detection markers, Clinical Cancer Research 12 (2006) 2117.

[11] X. S. Zhu, D. Y. Duan and N. G. Publicover, Magnetic bead based assay for C-reactive protein using quantum-dot fluorescence labeling and immunoaffinity separation, Analyst 135 (2010) 381.

[12] C. S. Lee, H. Lee and R. M. Westervelt, Microelectromagnets for the control of magnetic nanoparticles, Applied Physics Letters 79 (2001) 3308.

[13] B. B. Yellen, O. Hovorka and G. Friedman, Arranging matter by magnetic nanoparticle assemblers, Proceedings of the National Academy of Sciences of the United States of America 102 (2005) 8860.

[14] Q. Ramadan et al., Microcoils for transport of magnetic beads, Applied Physics Letters 88 (2006) 032501.

[15] C. X. Liu et al., On-chip separation of magnetic particles with different magnetophoretic mobilities, Journal of Applied Physics 101 (2007) 024913.

[16] R. J. S. Derks et al., Magnetic bead manipulation in a sub-microliter fluid volume applicable for biosensing, Microfluidics and Nanofluidics 3 (2007) 141. 
[17] G. Vieira et al., Magnetic Wire Traps and Programmable Manipulation of Biological Cells. Physical Review Letters 103 (2009) 128101.

[18] M. Donolato et al., On-Chip Manipulation of Protein-Coated Magnetic Beads via Domain-Wall Conduits, Advanced Materials 22 (2010) 2706.

[19] G. Ruan et al., Simultaneous Magnetic Manipulation and Fluorescent Tracking of Multiple Individual Hybrid Nanostructures, Nano Letters 10 (2010) 2220.

[20] T. Henighan et al., Manipulation of Magnetically Labeled and Unlabeled Cells with Mobile Magnetic Traps, Biophysical Journal 98 (2010) 412.

[21] L. Johansson et al., A magnetic microchip for controlled transport of attomole levels of proteins, Lab on a Chip $10(2010) 654$.

[22] M. T. Bryan et al., The effect of trapping superparamagnetic beads on domain wall motion, Applied Physics Letters 96 (2010) 192503.

[23] A. Hubert and R. Schäfer, Magnetic Domains, the analysis of magnetic microstructure, page 78, Springer, ISBN 3-540-64108-4 (1998).

[24] G. Vieira et al., Transport of magnetic microparticles via tunable stationary magnetic traps in patterned wires, Phys. Rev. B 85 (2012) 174440.

[25] A. Vansteenkiste and B. Van de Wiele, MuMax: a new high-performance micromagnetic simulation tool, J. Magn. Magn. Mater 323 (2011) 2585.

[26] J. Do, J. W. Choi and C. H. Ahn, Low-Cost Magnetic Interdigitated Array on a Plastic Wafer, IEEE Trans. Magnetics 40 (2004) 3009. 